\title{
Complexity and the Fractional Calculus
}

\author{
Pensri Pramukkul, ${ }^{1}$ Adam Svenkeson, ${ }^{1}$ Paolo Grigolini, ${ }^{1}$ Mauro Bologna, ${ }^{2}$ and Bruce West ${ }^{3}$ \\ ${ }^{1}$ Center for Nonlinear Science, University of North Texas, P.O. Box 311427, Denton, TX 76203-1427, USA \\ ${ }^{2}$ Instituto de Alta Investigación, Universidad de Tarapacá, Casilla 6-D, Arica, Chile \\ ${ }^{3}$ Information Science Directorate, Army Research Office, Research Triangle Park, NC 27709, USA
}

Correspondence should be addressed to Bruce West; bruce.j.west.civ@mail.mil

Received 21 March 2013; Accepted 28 March 2013

Academic Editor: Dumitru Baleanu

Copyright (c) 2013 Pensri Pramukkul et al. This is an open access article distributed under the Creative Commons Attribution License, which permits unrestricted use, distribution, and reproduction in any medium, provided the original work is properly cited.

\begin{abstract}
We study complex processes whose evolution in time rests on the occurrence of a large and random number of events. The mean time interval between two consecutive critical events is infinite, thereby violating the ergodic condition and activating at the same time a stochastic central limit theorem that supports the hypothesis that the Mittag-Leffler function is a universal property of nature. The time evolution of these complex systems is properly generated by means of fractional differential equations, thus leading to the interpretation of fractional trajectories as the average over many random trajectories each of which satisfies the stochastic central limit theorem and the condition for the Mittag-Leffler universality.
\end{abstract}

\section{Introduction}

The fractional calculus has developed in a number of significant ways in the recent past. Sokolov et al. [1] maintain that this calculus was restricted to the field of mathematics until the last decade of the twentieth century, when it became very popular among physicists as a powerful way to describe the dynamics of a variety of complex physical phenomena. For example, anomalous diffusion was described using fractional diffusion equations $[2,3]$; viscoelastic materials were modeled using fractional Langevin equations [4]; and complex dynamic systems could be governed using fractional control [5]. In the last decade the concept of fractional dynamics has gained further attention in the statistical and chemical physics communities [6]. Fractional differential equations have also been successfully applied to neural dynamics $[7,8]$ and ecology [9] as well as to traditional fields of engineering $[10,11]$ namely $[12,13]$.

Of particular interest to the authors is the growing literature on extending systems of nonlinear dynamic equations having strange attractor solutions to fractional nonlinear equations. Such extensions were typically made by replacing integer-valued derivatives by fractional derivatives; for example, in the Lorenz system [14-16], in the chaotic rigid body motion of gyros [17], in Hopfield-type neural networks [8], and in the immune model of HIV infection [18], to name a few. These replacements were made in attempts to incorporate dynamic mechanisms thought to be important that could not be captured by the traditional models, for example, complexity in the form of memory in time and nonlocality in space. The results of extending these nonlinear models has been to apparently introduce dissipation into the dynamics such that the solution on the strange attractor collapses to that of a stable fixed point. The appearance of a fixed point is interpreted to be a consequence of an induced dissipation mimicking the complexity modeled by the fractional derivatives.

Herein we provide an alternative interpretation of these extensions that involves the notion of a fluctuating trajectory and interpreting the fractional models as averages over an ensemble of these trajectories on the strange attractor. This view is consistent with one proposed as an extension of conservative Hamiltonian systems to fractional systems [19]. This generalization of classical mechanics is based on a randomization of chronological or clock time in the traditional phase space using the notion of operational time and subordination. Without going into the details of the extension of Hamilton's equations of motion to fractional form it suffices to note that 
fractional derivatives in chronological time are interpreted as averages of a particle's displacement and momentum over the fluctuating operational time. Consequently, the dynamics of a single fractional harmonic oscillator, for example, is considered to be an average over an ensemble of harmonic oscillators. Stanislavsky [19] emphasizes that each oscillator differs slightly from every other oscillator in frequency because of subordination. The phase space trajectories rather than being level energy curves instead spiral into the origin and the fractional oscillator "demonstrates a dissipative process stochastic by nature."

For the more general dynamical systems considered herein there is no Hamiltonian with which to generate the equations of motion. Recall that a strange attractor requires the system to dissipate energy. Therefore the approach taken here is stochastic rather than dynamic and requires the development of a new form of the central limit theorem (CLT), one that is compatible with the fractional calculus. Section 2 connects the familiar Mittag-Leffler function (MLF) solution to a Caputo fractional differential equation [20] to what we have named the stochastic central limit theorem (SCLT).

At the most elementary level the familiar relaxation rate equation is replaced by its fractional form

$$
\frac{d^{\alpha} \Psi(t)}{d t^{\alpha}}=-\lambda^{\alpha} \Psi(t)
$$

and here we interpret the fractional derivative to be of the Caputo form

$$
\frac{d^{\alpha} \Psi(t)}{d t^{\alpha}} \equiv \frac{1}{\Gamma(\alpha-n)} \int_{0}^{t} \frac{d t^{\prime}}{\left(t-t^{\prime}\right)^{\alpha+1-n}} \Psi^{(n)}\left(t^{\prime}\right),
$$

where the superscript is the derivative of integer order $n$ such that $n-1<\alpha<n$ and since $\alpha<1$ we have $n=1$. The solution to (1) is the MLF $[21,22]$

$$
\begin{aligned}
\Psi_{\mathrm{ML}}(t) & =E_{\alpha}\left(-(\lambda t)^{\alpha}\right) \\
& \equiv \sum_{n=0}^{\infty} \frac{(-1)^{n}}{\Gamma(1+n \alpha)}(\lambda t)^{n \alpha} \\
& = \begin{cases}1-\frac{(\lambda t)^{\alpha}}{\Gamma(1+\alpha)} \approx \exp \left[-\frac{(\lambda t)^{\alpha}}{\Gamma(1+\alpha)}\right], & \text { as } t \rightarrow 0 \\
\frac{1}{\Gamma(1-\alpha)(\lambda t)^{\alpha}}, & \text { as } t \rightarrow \infty\end{cases}
\end{aligned}
$$

Metzler and Klafter [23] exploited this connection between the stretched exponential and inverse power-law (IPL) distributions by interpreting the MLF as a survival probability and thereby establishing a bridge between the advocates of these two distinct forms of the survival probabilities as important signs of complexity.

In the Materials and Methods section we show that $\Psi_{\mathrm{ML}}(t)$ is universal, as had been advocated by Gorenflo and Mainardi [24] and others [25] in the same sense as the limit distributions in the CLTs of Laplace (CLT) and Lévy (generalized CLT) but here the universality is shown to be a consequence of the SCLT. In Section 2.1 the form of the SCLT is set up and in Section 2.2 the theorem is proven using a scaling argument. As a consequence of the SCLT we show in Section 2.3 by introducing fluctuating trajectories, interpreted as a proper representation of complex processes with memory, that the MLF is universal. The universality is a consequence of a subordination process. In the Results and Discussion section the connection between the Caputo fractional derivative and $\Psi_{\mathrm{ML}}(t)$ together with the fractional trajectories of Section 2, through subordination result in the fractional derivative being interpreted as an average over infinitely many random trajectories. In Section 4 we draw some conclusions.

\section{Materials and Methods}

In the late 1980s there was remarkable activity in the development of the theory of random summations, namely, the case where the number of summands is itself a random variable [26]. We adopt the term stochastic limit theorem rather than random summation to emphasize that we depart from the exemplary Poisson condition of [26]. The MLF is generated by a fluctuating number of events $m$ with fluctuations as large as the mean value $\langle m\rangle$. The main difference between the traditional and the SCLT is that the former rests on the sum of a fixed number $m$ of fluctuations, and on the rescaling procedure to use for $m \rightarrow \infty$. The SCLT is based on keeping fixed the probability $P_{S}$ of detecting an event, that is, the probability that an event is visible in an experiment. Each value of $P_{S}$ generates a sequence of $m$ elementary laminar regions but only one visible event at the end of the last laminar region. The SCLT focuses on the interval between two consecutive visible events and adopts a rescaling procedure to compensate for the incomplete-measurementinduced survival probability enhancement [27]. We show that all waiting-time $p d f$ 's generating a non-integrable survival probability as a consequence of the SCLT yield $\psi_{\mathrm{ML}}(t)$. This proof leads us to conclude that $\Psi_{\mathrm{ML}}(t)$ is universal.

2.1. Stochastic Central Limit Theorem. The concept of survival probability is connected to the stochastic perspective of a complex system generating events in time. The time interval between two consecutive events (laminar region) is assigned the values \pm 1 , according to a coin tossing prescription [28]. At time $t=0$ the system is prepared by selecting all the realizations with an event occurring at that time, with ensuing positive laminar regions. As a consequence the probability that no event occurs up to time $t$, denoted as $\Psi(t)$, is properly termed a survival probability, and the function

$$
\psi(t) \equiv-\frac{d \Psi(t)}{d t}
$$

is the waiting-time probability density function $(p d f)$. We adopt the symbols $\Psi_{\mathrm{ML}}(t)$ and $\psi_{\mathrm{ML}}(t)$ to denote the MLF survival probability and the corresponding waiting-time $p d f$, respectively. Physical examples of $\psi_{\mathrm{ML}}(t)$ generated by the cooperative interaction of many units can be found [27, 29], with the important observation that the stretched exponential 
regime becomes more extended if the probability of generating a visible cooperative event decreases as discussed in Section 2.2.

We assume that the time interval between two consecutive critical events generated by the complex system under study is given by the waiting-time $p d f$

$$
\psi(\tau) \propto \frac{1}{\tau^{\mu}}, \quad \text { with } 1<\mu<2 .
$$

The corresponding cumulative distribution $\Psi(\tau)$ has the form

$$
\lim _{\tau \rightarrow \infty} \Psi(\tau) \propto \frac{1}{\tau^{\alpha}}, \quad \text { with } \alpha \equiv \mu-1<1 .
$$

The origin of this condition, usually interpreted as a manifestation of complexity, can either be the anomalous nature of the dynamics under investigation $[30,31]$ or the condition of criticality [32]. In the former case the property described by (7) can, for example, be the consequence of diffusing molecules being trapped for long times in wells with a random distribution of depths. In the latter and less well known situation the emergence of temporal complexity is due to the cooperative action of many interacting units. At the onset of the cooperation-induced phase transition from disorder to order, the mean field fluctuates and its nonstationary waiting-time $p d f$ corresponds to an IPL $\psi(t)$ [33].

We adopt for the Laplace transform of the time function $f(t)$ the following notation:

$$
\widehat{f}(u)=\mathfrak{E}\{f(t) ; u\}=\int_{0}^{\infty} d t \exp (-u t) f(t) .
$$

It is important to stress that to satisfy the long-time limit of (7) the Laplace transform of $\psi(\tau)$ has the functional form

$$
\widehat{\psi}(u)=1-\left(\frac{u}{\lambda_{0}}\right)^{\alpha}+\Xi(u),
$$

with the condition on the subsidiary function $\Xi(u)$

$$
\lim _{u \rightarrow 0} \lambda_{0}^{\alpha} \frac{\Xi(u)}{u^{\alpha}}=0
$$

Therefore the subsidiary function must vanish more rapidly than $u^{\alpha}$ as $u \rightarrow 0$.

Note that the Laplace transform of the MLF survival probability given by $(3)$ is $[20,22]$

$$
\widehat{\Psi}_{\mathrm{ML}}(u)=\frac{u^{\alpha-1}}{u^{\alpha}+\lambda_{0}^{\alpha}}
$$

and the relation to the Laplace transform of the waiting-time $p d f$ is

$$
\widehat{\Psi}_{\mathrm{ML}}(u)=\frac{1-\widehat{\psi}_{\mathrm{ML}}(u)}{u}
$$

so that with a little algebra we obtain

$$
\widehat{\psi}_{\mathrm{ML}}(u)=\frac{1}{\left(u / \lambda_{0}\right)^{\alpha}+1},
$$

thereby yielding for the subsidiary function in (10)

$$
\Xi(u)=\left(\frac{u}{\lambda_{0}}\right)^{2 \alpha} \frac{1}{1+\left(u / \lambda_{0}\right)^{\alpha}},
$$

satisfying the condition of (11). In other words, the properties of (10) and (11) are fulfilled by all the waiting-time $p d f$ 's with the scale-free condition of (7). We now show that the waitingtime $p d f$ corresponding to the sum of a large numbers of times each of which is generated by the generic $p d f \psi(t)$ of (7), not necessarily of the MLF type, are MLF waiting-time pdf's.

2.2. Imperfect Detection of Events. To make this demonstration as clear as possible and at the same time provide an intuitive understanding of the SCLT, let us imagine that the detector used to monitor the events produced by (7) is not very accurate and that the probability of perceiving these events is

$$
P_{S}<1
$$

As a consequence of this imperfection a time $t$ between two consecutive visible events is the sum of $m$ elementary times derived from the condition

$$
P(m)=P_{S}\left(1-P_{S}\right)^{m}
$$

which is the probability that the first $m$ events after the initial preparation event are not visible while the $(m+1)$ th event is visible. For $P_{S} \rightarrow 0$,

$$
P(m)=P_{S} \exp \left(-m P_{S}\right),
$$

thereby implying that the standard deviation is on the same order as the mean

$$
\frac{\left(\left\langle m^{2}\right\rangle-\langle m\rangle^{2}\right)}{\langle m\rangle^{2}} \approx 1 \text {. }
$$

Thus, the condition

$$
\langle m\rangle=\frac{1}{P_{S}} \longrightarrow \infty
$$

of the SCLT is quite different from the condition $m \rightarrow \infty$ of the Gauss and Lévy CLTs, since $m$ has very large fluctuations around $\langle m\rangle$ in the traditional argument. To better understand the new theorem, we note that the probability of generating at time $t$ an event that is the last of a sequence of $m$ events occurring at earlier times, $\psi_{m}(t)$, does not satisfy the condition of generating a MLF stable form for $m \rightarrow \infty$. However, the waiting-time $p d f$ of the time intervals between visible events, does, for $P_{S} \rightarrow 0$. The $p d f$ of finding a visible event a time interval $t$ after an earlier visible event is given by

$$
\psi_{V}(t)=\sum_{m=0}^{\infty} P_{S}\left(1-P_{S}\right)^{m} \psi_{m+1}(t) .
$$


Implementing the well known property for renewal processes $[22,28]$

$$
\widehat{\psi}_{m}(u)=[\widehat{\psi}(u)]^{m}
$$

we obtain from the Laplace transform of (21) after a little algebra

$$
\widehat{\psi}_{V}(u)=\frac{\widehat{\psi}(u)}{1-\left(\left(1-P_{S}\right) / P_{S}\right)(\widehat{\psi}(u)-1)} .
$$

Using the partitioning of (10) we rewrite (23) in the more convenient form

$$
\widehat{\psi}_{V}(u)=\frac{1-\left(u / \lambda_{0}\right)^{\alpha}+\Xi(u)}{1+\left(\left(1-P_{S}\right) / P_{S}\right)\left(u / \lambda_{0}\right)^{\alpha}-\left(\left(1-P_{S}\right) / P_{S}\right) \Xi(u)} .
$$

To fulfill the limiting condition of (11) the slowest contribution to $\Xi(u)$ must be

$$
\Xi_{\text {slowest }}(u)=k u^{\alpha+\epsilon},
$$

with $\epsilon>0$. Rescaling the Laplace variable $u$ with the detection probability

$$
u=u^{\prime} P_{S}^{1 / \alpha}
$$

transforms (24) into

$$
\widehat{\psi}_{V}\left(u^{\prime}\right)=\frac{1-P_{S}\left(u^{\prime} / \lambda_{0}\right)^{\alpha}+\Xi\left(u^{\prime} P_{S}{ }^{1 / \alpha}\right)}{1+\left(1-P_{S}\right)\left(u^{\prime} / \lambda_{0}\right)^{\alpha}-\left(\left(1-P_{S}\right) / P_{S}\right) \Xi\left(u^{\prime} P_{S}{ }^{1 / \alpha}\right)}
$$

that for $P_{S} \rightarrow 0$ reduces to

$$
\widehat{\psi}_{V}\left(u^{\prime}\right)=\frac{1}{1+\left(u^{\prime} / \lambda_{0}\right)^{\alpha}}
$$

In fact, the rescaled slowest contribution to $\Xi(u)$ is proportional to $P_{S}^{1+\epsilon / \alpha}$ thereby making the contributions of $\Xi(u)$ vanish for $P_{S} \rightarrow 0$ in both the numerator and the denominator of (27).

We see that after rescaling $\widehat{\psi}_{V}(u)$ coincides with (14) and consequently its inverse Laplace transform is

$$
\psi_{V}(t)=-\frac{d E_{\alpha}\left(-\left(\lambda_{0} t\right)^{\alpha}\right)}{d t} .
$$

Consequently, the survival probability of the visible events is the MLF. This is the essence of the SCLT.

2.3. Fluctuating Trajectories. To use the SCLT to interpret the fractional trajectories as averages over infinitely many stochastic realizations, it is convenient to get $\Psi_{\mathrm{ML}}(t)$ from the survival probability of the time interval between two consecutive visible events. We note from (13) that

$$
\widehat{\Psi}_{V}(u)=\frac{1-\widehat{\psi}_{V}(u)}{u} .
$$

It is straightforward to show that inserting $\widehat{\psi}_{V}(u)$ of (23) into (30) yields a result equivalent to

$$
\Psi_{V}(t)=\sum_{n=0}^{\infty} \int_{0}^{t} d t^{\prime} \psi_{n}\left(t^{\prime}\right) \Psi\left(t-t^{\prime}\right)\left(1-P_{S}\right)^{n}
$$

which has the well known Montroll-Weiss continuous time random walk (CTRW) structure [34]. The Laplace transform of $\Psi_{V}(t)$ from (31) reads

$$
\widehat{\Psi}_{V}(u)=\frac{1}{u+\widehat{\Phi}(u) P_{S}},
$$

where $\widehat{\Phi}(u)$ is the Laplace transform of the Montroll-Weiss memory kernel defined by

$$
\widehat{\Phi}(u)=\frac{u \widehat{\psi}(u)}{1-\widehat{\psi}(u)} .
$$

Note that by using inverse Laplace transforms it is straightforward to establish that (31) is equivalent to

$$
\frac{d}{d t} \Psi_{V}(t)=-P_{S} \int_{0}^{t} d t^{\prime} \Phi\left(t^{\prime}\right) \Psi_{V}\left(t-t^{\prime}\right),
$$

whose time convolution structure justifies the adoption of the term memory kernel for $\Phi(t)$.

Due to the equivalence between (21) and (31) we can use the $P_{S}$ scaling argument to immediately conclude that

$$
\lim _{P_{S} \rightarrow 0} \widehat{\Psi}_{V}\left(u^{\prime}\right)=\frac{1}{u^{\prime}+u^{\prime 1-\alpha} \lambda_{0}^{\alpha}},
$$

thereby demonstrating why the MLF is ubiquitous in data sets since it is the distribution of visible, that is, measurable events.

To shed further light into the SCLT, let us notice that the condition $P_{S} \rightarrow 0$ has the effect of turning $n$ into a virtually continuous time $\tau,\left(1-P_{S}\right)^{n}$ into $\exp \left(-P_{S} \tau\right)$ and $\Psi_{V}(t)$ of (31) into $E_{\alpha}\left(-P_{S}\left(\lambda_{0} t\right)^{\alpha}\right)$. Thus the MLF survival probability with $\lambda=\left(P_{S}\right)^{1 / \alpha} \lambda_{0}$ is the counterpart in real time $t$ of the ordinary exponential function $\exp \left(-P_{S} \tau\right)$,

$$
E_{\alpha}\left(-P_{S}\left(\lambda_{0} t\right)^{\alpha}\right)=\int_{0}^{\infty} d \tau p^{(S)}(t, \tau) \exp \left(-P_{S} \tau\right)
$$

and $p^{(S)}(t, \tau)$ is the $p d f$ of times $t$ corresponding to the continuous time $\tau$. A straightforward way of deriving (36) from (31) rests on observing that the Laplace transform of (31), namely, (32), can be interpreted as a double Laplace transform $p^{(S)}(u, s)$ with $s=P_{S}$. By inverse Laplace transforming $p^{(S)}\left(u, s=P_{S}\right)$ with respect to $u$ we obtain (36) in accordance with [35-37]. The time $t$ can be interpreted as a diffusing position of a random walker that keeps jumping in the same direction. It is, in fact, an asymmetric Lévy process [35-37]. We are therefore led to interpret the SCLT as a consequence of the generalized CLT of Lévy. The condition $n \rightarrow \infty$ generates the Lévy stable $p d f$ and the sum over infinitely many Lévy processes weighted by the exponential function $\exp \left(-P_{S} \tau\right)$ generates the MLF survival probability, in accordance with earlier results $[38,39]$. 


\section{Results and Discussion}

The SCLT is the first important result of this paper. The traditional CLT yields the Normal distribution as the limit $p d f$. The generalized CLT developed by Lévy yields the $\alpha$-stable distribution as the limit $p d f$. Finally the SCLT presented herein yields the MLF as the limit $p d f$. On the basis of the SCLT we established a physical interpretation of fractional trajectories that had not been previously considered in the literature for non-conservative dynamic systems. The MLF survival probability can be interpreted as the realization in the continuous time $t$ of the traditional exponential relaxation process

$$
\frac{d \Psi(\tau)}{d \tau}=-P_{S} \Psi(\tau)
$$

in operational time. The probabilistic structure of (36) represents the sum over infinitely many such random relaxation processes, which turns out to be equivalent to the MLF survival probability. In the same way a fractional trajectory, not necessarily dissipative, was proven to be a sum over infinitely many stochastic trajectories that for historical reasons we call Montroll-Weiss trajectories.

3.1. Subordination and Fractional Dynamics. To relate the physical interpretation established in the previous section to the replacement fractional differential equations we introduce the nomenclature of a fractional trajectory. Consider the differential equation in operational time

$$
\frac{d}{d \tau} \mathbf{V}(\tau)=-\widetilde{\Gamma} \mathbf{V}(\tau)
$$

where $\mathbf{V}$ is a generic multidimensional vector and $\widetilde{\Gamma}$ is a generic operator either linear or nonlinear acting on the components of the vector $\mathbf{V}$. With this concise notation we may describe, for instance, the Lorenz system [14-16], which is a remarkable application of the fractional calculus to chaos, with $\mathbf{V}=\{X, Y, Z\}$. All the studies using the fractional calculus can be represented by the notation of (38). Consequently, in the nonlinear systems whose dynamics unfold on a strange attractor we follow the tradition and replace the integer order derivative $d / d \tau$ with the Caputo fractional derivative of index $\alpha$, with $\alpha<1$, to obtain

$$
\frac{d^{\alpha}}{d t^{\alpha}} \mathbf{V}(t)=-\tilde{\Gamma} \mathbf{V}(t)
$$

We refer to the solution of (39) as a fractional trajectory, that being the time trace of the solution in phase space for the system. Here we anticipate the second result of this paper that being the time evolution of $\mathbf{V}(t)$, the fractional trajectory, is an average over infinitely many stochastic Montroll-Weiss trajectories.

To properly define a Montroll-Weiss trajectory, let us go back to (38) and adopt a numerical procedure to integrate it over the operational time and obtain

$$
\mathbf{V}(\tau+\Delta \tau)=(1-\Delta \tau \widetilde{\Gamma}) \mathbf{V}(\tau)
$$

The numerical procedure replaces the continuous one with a discrete time as would be appropriate in a numerical algorithm. Thus we can write for the $n$th iterate of this equation, using the notation $\mathbf{V}(n) \equiv \mathbf{V}(n \Delta \tau)$ :

$$
\mathbf{V}(n)=(1-\Delta \tau \widetilde{\Gamma})^{n} \mathbf{V}(0)
$$

Note that the numerical solution of (38) realized by means of the prescription (41) is an extension to a generic trajectory of the exponential relaxation $\left(1-P_{S}\right)^{n}$. For this reason the condition of perfect integration $\Delta \tau \rightarrow 0$ corresponds to $P_{S} \rightarrow 0$, thereby establishing a connection with the SCLT.

A stochastic Montroll-Weiss trajectory is obtained by assuming the transition $\mathbf{V}(n) \rightarrow \mathbf{V}(n+1)$ is a crucial event occurring at the time $t(n)=\tau_{1}+\cdots \tau_{n}$, where the $\tau_{i}$ are random times with the waiting-time $p d f \psi(\tau)$ of (7), not necessarily identical to $\psi_{\mathrm{ML}}(\tau)$. We assign to $\mathbf{V}(t)$ the value $\mathrm{V}(n)$ with $n$ fulfilling the condition $t(n) \leq t<t(n+1)$.

It is important to restate the difference between the Lévy CLT and the SCLT. As shown in [30], the $p d f p^{(S)}(t, \tau)$ is an asymmetric Lévy process, which ought not to be confused with the stable $\psi_{\mathrm{ML}}(t)$ generated by the procedure of this paper. An average over infinitely many Montroll-Weiss trajectories yields

$$
\mathbf{V}(t)=\sum_{n=0}^{\infty} \int_{0}^{t} d t^{\prime} \psi_{n}\left(t^{\prime}\right) \Psi\left(t-t^{\prime}\right)(1-\Delta \tau \widetilde{\Gamma})^{n} \mathbf{V}(0)
$$

The left hand side of (42) is a Gibbs ensemble average over infinitely many fluctuating trajectories and should be denoted by the symbol $\langle\mathbf{V}(t)\rangle$. However to stress its connection with the fractional derivative formalism we continue to use the symbol $\mathbf{V}(t)$. The Laplace transform of $\mathbf{V}(t)$ is

$$
\widehat{\mathbf{V}}(u)=\frac{1}{u+\widehat{\Phi}(u) \Delta \tau \widetilde{\Gamma}} \mathbf{V}(0) .
$$

On the basis of the SCLT, we conclude that for $\Delta \tau \rightarrow 0$, corresponding to $P_{S} \rightarrow 0$,

$$
\widehat{\mathbf{V}}(u)=\frac{1}{u+u^{1-\alpha} \lambda_{0}^{\alpha} \Delta \tau \widetilde{\Gamma}} \mathbf{V}(0) .
$$

This allows us to replace the average given by (42) with its fractional differential equivalent

$$
\frac{d^{\alpha}}{d t^{\alpha}} \mathbf{V}(t)=-\Delta \tau \lambda_{0}^{\alpha} \widetilde{\Gamma} \mathbf{V}(t)
$$

In fact, using the Caputo fractional derivatives we have

$$
£\left\{\frac{d^{\alpha}}{d t^{\alpha}} \mathbf{V}(t) ; u\right\}=u^{\alpha} \widehat{\mathbf{V}}(u)-u^{\alpha-1} \mathbf{V}(0) .
$$

Laplace transforming (45) with this rule has the effect of yielding (44), thereby establishing that the fractional differential equation given by (39) is equivalent to the mean MontrollWeiss trajectory of (42), under the condition $\lambda_{0}^{\alpha} \Delta \tau=1$. Note that $\Delta \tau$ must be small enough as to ensure a convergent solution to (38) with the possible effect of making $\lambda_{0}$ so large 
as to virtually cancel the stretched exponential regime of the MLF. With $\lambda_{0}^{\alpha} \Delta \tau=1$ the effective rate $\lambda$ is determined by the eigenvalues of $\widetilde{\Gamma}$, which determine the density of events, thereby making the stretched exponential become ostensible with very low event densities, in accordance again with the property of incomplete measurement [27].

\section{Conclusions}

We have established the universality of the MLF survival probability using a SCLT. The term stochastic in SCLT emphasizes the fact that the large number of elementary laminar times whose sum generates the time interval between two consecutive visible events is a widely fluctuating number with a fluctuation intensity as large as the mean number of events involved in the process. The SCLT leads to a new perspective of fractional trajectories, which yields a new physical interpretation of their claimed stronger stability on the part of those that have replaced the integer with fractional derivatives. The dissipative nature of the fractional trajectories has to be interpreted as a form of phase decorrelation process rather than one with friction. The fractional version of the popular Lotka-Volterra ecological dynamics, for instance, generates a fractional trajectory that is the sum over infinitely many Montroll-Weiss trajectories. Each Montroll-Weiss trajectory is an ordinary Lotka-Volterra cycle in the operational time $n$. Transitioning from the operational time $n$ to the chronological time $t$ spreads these trajectories over the entire Lotka-Volterra cycle thereby generating the mistaken impression that the resulting average trajectory reaches equilibrium through a dissipative process.

\section{Acknowledgments}

M. Bologna acknowledges financial support from FONDECYT Project no. 1110231. The authors warmly thank Welch and ARO for the financial support of this work through Grant no. B-1577 and Grant no. W911NF1110478, respectively.

\section{References}

[1] I. M. Sokolov, J. Klafter, and A. Blumen, "Fractional kinetics," Physics Today, vol. 55, no. 11, pp. 48-54, 2002.

[2] V. Seshadri and B. J. West, "Fractal dimensionality of Lévy processes," Proceedings of the National Academy of Sciences of the United States of America, vol. 79, no. 14, pp. 4501-4505, 1982.

[3] R. Metzler and J. Klafter, "The random walk's guide to anomalous diffusion: a fractional dynamics approach," Physics Reports, vol. 339, no. 1, p. 77, 2000.

[4] R. Metzler, W. G. Glöke, and T. F. Nonnenmacher, "Fractional model equation for anomalous diffusion," Physica A, vol. 211, no. 1, pp. 13-24, 1994.

[5] I. Podlubny, Fractional Differential Equations, Academic Press, New York, NY, USA, 1999.

[6] J. Klafter, S. C. Lim, and R. Metzler, Eds., Fractional Dynamics: Recent Advances, World Scientific, Singapore, 2012.

[7] B. N. Lundstrom, M. H. Higgs, W. J. Spain, and A. L. Fairhall, "Fractional differentiation by neocortical pyramidal neurons," Nature Neuroscience, vol. 11, no. 11, pp. 1335-1342, 2008.
[8] E. Kaslik and S. Sivasundaram, "Nonlinear dynamics and chaos in fractional-order neural networks," Neural Networks, vol. 32, pp. 245-256, 2012.

[9] E. Ahmed, A. M. A. El-Sayed, and H. A. A. El-Saka, "Equilibrium points, stability and numerical solutions of fractionalorder predator-prey and rabies models," Journal of Mathematical Analysis and Applications, vol. 325, no. 1, pp. 542-553, 2007.

[10] R. L. Magin, Fractional Calculus in Bioengineering, Begell House, Redding, Conn, USA, 2006.

[11] F. Gómez, J. Bernal, J. Rosales, and T. Cordova, "Modeling and simulation of equivalent circuits in description of biological systems-a fractional calculus approach," Journal of Electrical Bioimpedance, vol. 3, p. 2, 2012.

[12] D. Baleanu, K. Diethelm, E. Scalas, and J. J. Trujillo, Fractional Calculus Models and Numerical Methods, vol. 3 of Complexity, Nonlinearity and Chaos, World Scientific, 2012.

[13] S. Bhalekar, V. Daftardar-Gejii, D. Baleanu, and R. Magin, "Transient chaos in fractional Bloch equations," Computers \& Mathematics with Applications, vol. 64, no. 10, pp. 3367-3376, 2012.

[14] I. Grigorenko and E. Grigorenko, "Chaotic dynamics of the fractional Lorenz system," Physical Review Letters, vol. 91, no. 3, Article ID 034101, 4 pages, 2003.

[15] C. Li and G. Chen, "Chaos in the fractional order Chen system and its control," Chaos, Solitons and Fractals, vol. 22, no. 3, pp. 549-554, 2004.

[16] R. W. Ibrahim, "Stability and stabilizing of fractional complex Lorenz Systems," Abstract and Applied Analysis, vol. 2013, Article ID 127103, 13 pages, 2013.

[17] L.-J. Sheu, H.-K. Chen, J.-H. Chen et al., "Chaos in the Newton-Leipnik system with fractional order," Chaos, Solitons \& Fractals, vol. 36, no. 1, pp. 98-103, 2008.

[18] H. Ye and Y. Ding, "Nonlinear dynamics and chaos in a fractional-order HIV model," Mathematical Problems in Engineering, vol. 2009, Article ID 378614, 12 pages, 2009.

[19] A. A. Stanislavsky, "Hamiltonian formalism of fractional systems," The European Physical Journal B, vol. 49, no. 1, pp. 93-101, 2006.

[20] F. Mainardi and R. Gorenflo, "On Mittag-Leffler-type functions in fractional evolution processes," Journal of Computational and Applied Mathematics, vol. 118, no. 1-2, pp. 283-299, 2000.

[21] M. G. Mittag-Leffler, "Sur la nouvelle function E $\alpha(\mathrm{x})$," Comptes Rendus De L’Académie Des Sciences, vol. 137, pp. 554-558, 1903.

[22] B. J. West, M. Bologna, and P. Grigolini, Physics of Fractal Operators, Springer, New York, NY, USA, 2003.

[23] R. Metzler and J. Klafter, "From stretched exponential to inverse power-law: fractional dynamics, Cole-Cole relaxation processes, and beyond," Journal of Non-Crystalline Solids, vol. 305, no. 1-3, pp. 81-87, 2002.

[24] R. Gorenflo and F. Mainardi, "Continuous time random walk, Mittag-Leffler waiting time and fractional diffusion: mathematical aspect," in WEHeraeus-Seminar on Anomalous Transport: Experimental Results and Theoretical Challenges, Physikzentrum Bad-Honnef, July 2006.

[25] M. M. Meerschaert, E. Nane, and P. Vellaisamy, "The fractional Poisson process and the inverse stable subordinator," Electronic Journal of Probability, vol. 16, pp. 1600-1620, 2011.

[26] B. V. Gnedenko and V. Yu. Korolev, Random Summation: Limit Theorems and Applications, CRC Press, New York, NY, USA, 1996. 
[27] M. Zare and P. Grigolini, "Cooperation in neural systems: bridging complexity and periodicity," Physical Review E, vol. 86, Article ID 051918, 6 pages, 2012.

[28] G. Zumofen and J. Klafter, "Scale-invariant motion in intermittent chaotic systems," Physical Review E, vol. 47, no. 2, pp. 851863, 1993

[29] R. Failla, P. Grigolini, M. Ignaccolo, and A. Schwettmann, "Random growth of interfaces as a subordinated process," Physical Reviews E, vol. 70, Article ID 010101, 4 pages, 2004.

[30] J. P. Bouchaud, "Weak ergodicity breaking and aging in disordered systems," Journal De Physique I, vol. 2, no. 9, pp. 1705-1713, 1992.

[31] E. Barkai, Y. Garini, and R. Metzler, "Strange kinetics of single molecules in living cells," Physics Today, vol. 65, no. 8, p. 29, 2012.

[32] E. Tagliazucchi, P. Balenzuela, D. Fraiman, and D. R. Chialvo, "Criticality in large-scale brain fMRI dynamics unveiled by a novel point process analysis," Frontiers in Physiology, vol. 3, article 15, 2012.

[33] M. Turalska, B. J. West, and P. Grigolini, “Temporal complexity of the order parameter at the phase transition," Physical Reviews E, vol. 83, Article ID 061142, 6 pages, 2011.

[34] E. W. Montroll and G. H. Weiss, "Random walks on lattices. II," Journal of Mathematical Physics, vol. 6, pp. 167-181, 1965.

[35] J.-P. Bouchaud and A. Georges, "Anomalous diffusion in disordered media: statistical mechanisms, models and physical applications," Physics Reports, vol. 195, no. 4-5, pp. 127-293, 1990.

[36] H. C. Fogedby, "Lévy flights in random environments," Physical Review Letters, vol. 73, pp. 2517-2520, 1994.

[37] H. C. Fogedby, "Lévy flights in quenched random force fields ," Physical Reviews E, vol. 58, pp. 1690-1712, 1998.

[38] N. H. Bingham, "Limit theorems for occupation times of Markov processes," Zeitschrift für Wahrscheinlichkeitstheorie und Verwandte Gebiete, vol. 17, no. 1, pp. 1-22, 1971.

[39] A. A. Stanislavsky, "Fractional dynamics from the ordinary Langevin equation," Physical Review E, vol. 67, no. 2, Article ID 021111, 6 pages, 2003. 


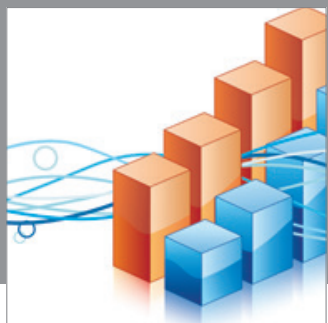

Advances in

Operations Research

mansans

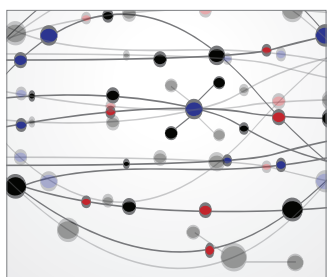

The Scientific World Journal
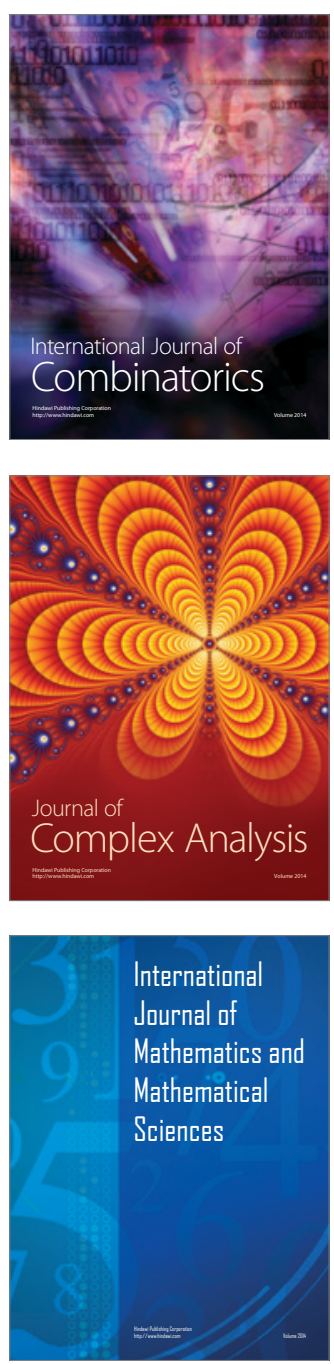
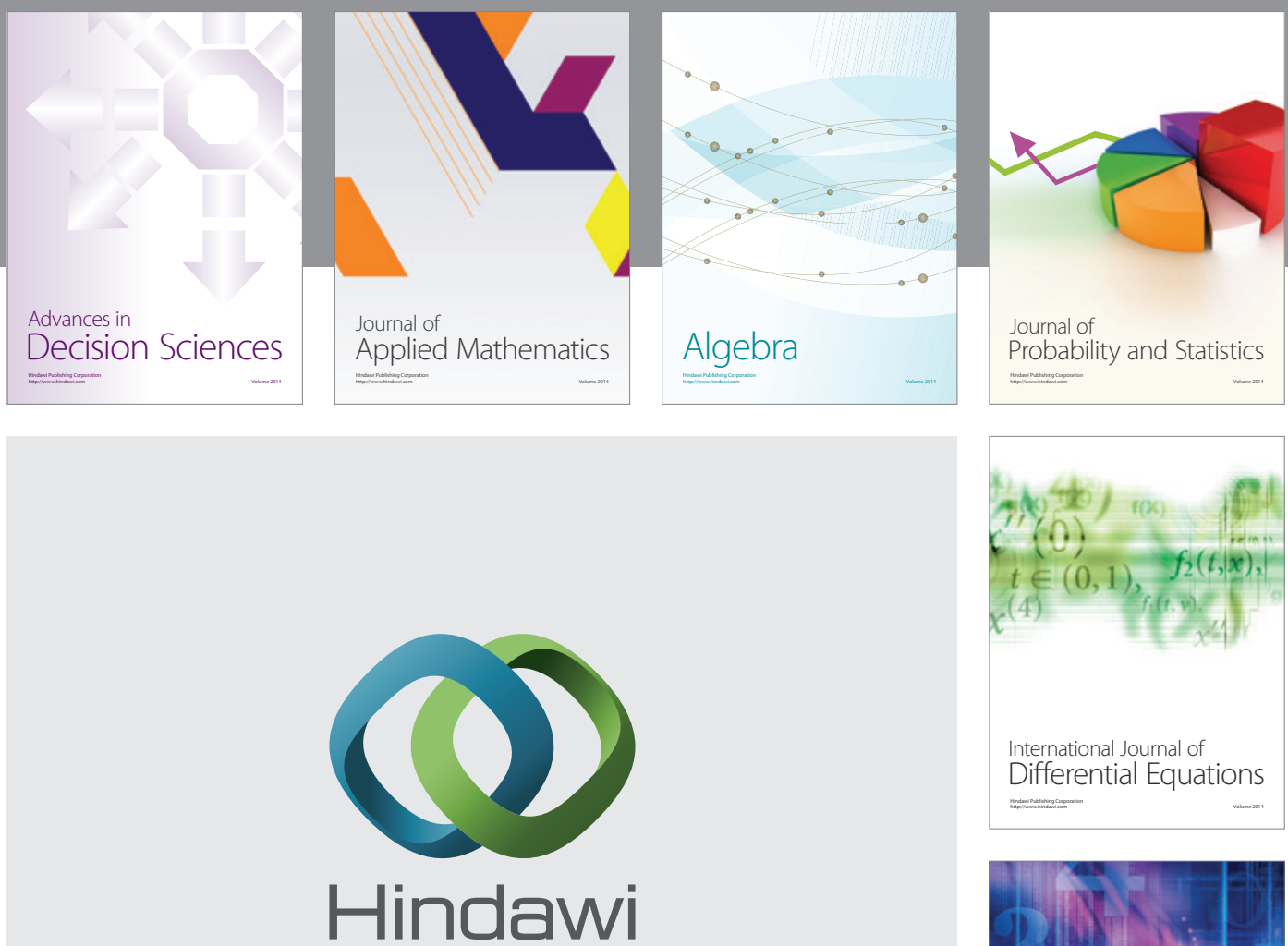

Submit your manuscripts at http://www.hindawi.com
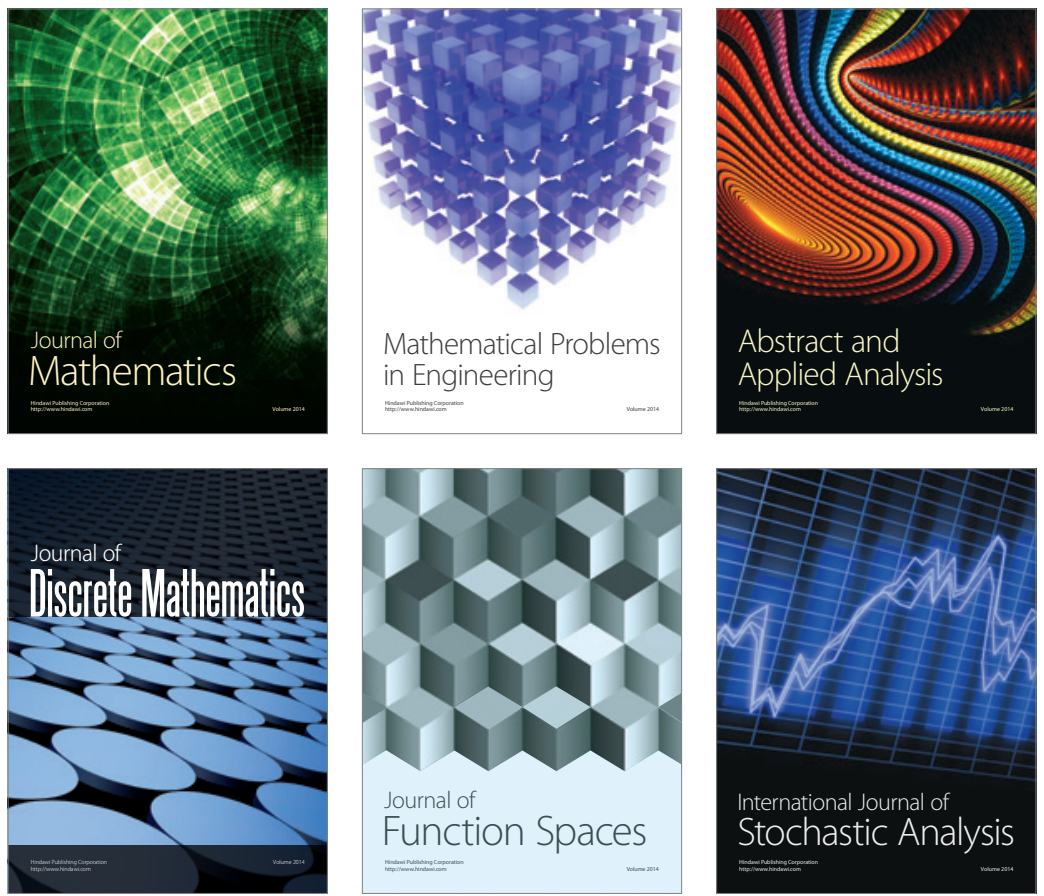

Journal of

Function Spaces

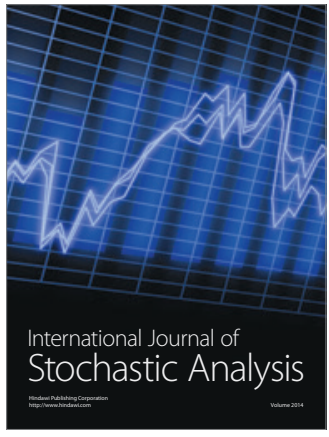

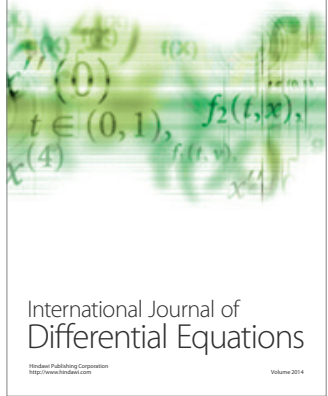
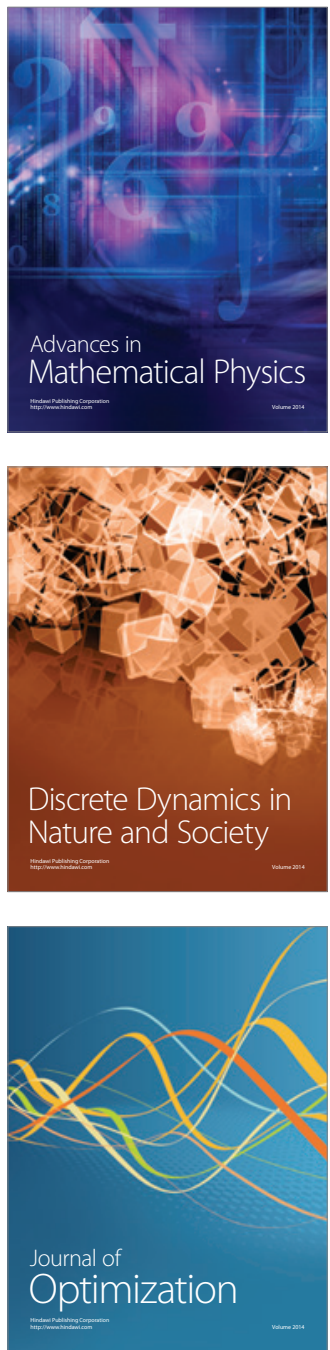